\title{
Chagas disease reactivation: cutaneous manifestations in a transplanted patient*
}

\author{
Maria Guillermina Ferraressoํㅜ, Ana Clara Torre ${ }^{1}$, Maria Manuela Martínez Piva ${ }^{1}$, Laura Barcan ${ }^{2}$
}

DOI: http:/ / dx.doi.org/10.1590/abd1806-4841.20187855

\begin{abstract}
Chagas disease is an endemic zoonosis caused by a protozoan agent called Trypanosoma cruzi. It is mainly transmitted by a hematophagous vector, and less frequently by blood transfusion, transplacental and solid organ transplant. In most cases, primary infection is not diagnosed and the disease progresses to a chronic phase. Immunosuppressed patients are a vulnerable population that may present an acute, atypical and severe reactivation of the chronic form of this disease. We hereby report a case of a female patient, who received a renal transplant with immunosuppressive treatment, who was diagnosed with a chagasic hypodermitis secondary to an acute reactivation of a chronic phase of this disease. We describe the clinical features, epidemiological and histopathological findings, treatment and course.
\end{abstract}

Keywords: Chagas disease; Immunosuppression; Kidney transplantation; Panniculitis

\section{INTRODUCTION}

Chagas disease is a histiocytic zoonosis caused by a flagellated protozoan called Trypanosoma cruzi. It is mainly transmitted by a hematophagous vector called Triatoma infestans. It has nocturnal habits and acquires the trypomastigotes feeding off a wild, domestic animal, or an infected human.

The trypomastigotes multiply in the midgut of the arthropod as epimastigotes. Transmission to the new host occurs when the parasite-laden feces contaminate enter by the bite wound, conjunctiva or other surfaces. ${ }^{1}$ From there, they reach the bloodstream and can infect any nucleated cells, with predilection for the heart, brain and gastrointestinal system. There, the parasites become intracellular, reproduce by binary fission as amastigotes, when they can hatch the cell and reach the blood circulation again, infecting other cells. ${ }^{2}$

The acute infection is often asymptomatic, but patients can occasionally develop fever, asthenia, gastrointestinal symptoms or cardiac involvement (myocarditis, cardiomegaly, congestive heart failure and arrhythmias). An asymptomatic erythematous-violet nodule, known as "chagoma" may appear at the site of inoculation. When the entrance door is the conjunctiva, the ophthalmoganglionar complex or Romaña's sign could be observed and is characterized by unilateral bi-palpebral edema, conjunctivitis, and regional adenopathy. $^{2}$

A later manifestation of the acute stage is a morbilliform rash known as "trypanosomides". It is asymptomatic and usually appears a few weeks after the inoculation. ${ }^{3}$ In the acute phase, the diagnosis is achieved by direct methods as microhematocrit, Strout or PCR of peripheral blood. Blood culture, the thick drop test and xenodiagnosis are helpful if they are performed in specialized medical centers. ${ }^{4}$ In most patients, the acute phase resolved in a few months, and parasitemia levels become negative. The acute phase

Received 13 November 2017.

Accepted 18 January 2018.

* Work conducted at Hospital Italiano de Buenos Aires, Buenos Aires, Argentina.

Financial support: None.

Conflict of interest: None.

Department of Dermatology, Hospital Italiano de Buenos Aires, Buenos Aires, Argentina.

Infectology section, Department of Internal Medicine, Hospital Italiano de Buenos Aires, Buenos Aires, Argentina.

MaILING AdDRess:

Maria Guillermina Ferraresso

E-mail: maria.ferraresso@hospitalitaliano.org.ar

@ 2018 by Anais Brasileiros de Dermatologia

(cc) BY-NC 
is not frequently diagnosed, and the disease progresses to an indeterminate or a chronic stage where the amastigotes remain quiet inside the organs' cells. ${ }^{5}$ The difference among these stages is that, while in chronic disease there is evidence of disease (cardiomegaly, megacolon, megaesophagus or hypodermitis) in the indeterminate one there is not. ${ }^{6}$

There is a usual balance between immunity and infection during an indeterminate or chronic stage. ${ }^{7}$ When the immunity is affected, this balance is broken and the infection reappears mainly affecting the heart, brain and hypodermis. ${ }^{6}$ In the latter, a localized or extensive hypodermitis can occur, as well as lesions like erythema nodosum or cellulitis. ${ }^{5,8}$

\section{CASE REPORT}

This was a 46-year-old woman, from a non-rural area of Córdoba (Argentina), who had type 2 diabetes and chronic renal failure. She underwent a renal transplant and immunosuppressive treatment with prednisone $8 \mathrm{mg} /$ day, tacrolimus $5 \mathrm{mg} /$ day and mycophenolate $720 \mathrm{mg} /$ day. In the pre-transplant studies Chagas positive serology was detected (ELISA and indirect haemagglutination). Three months later she developed fever, asthenia and pain in both limbs. The physical examination showed multiple painful, erythematous, infiltrated plaques, with diffuse borders, irregular size and shape, with increase of the local temperature, extended on the both gluteus, thighs, legs and left arm (Figure 1 and 2). With the suspicion of infectious or inflammatory hypodermitis, we performed surgical skin biopsies for histopathology, culture and Chagas PCR. Peripheral blood and tissue Chagas PCR were positive (12 and 76785 eq.p/mL respectively). Histological examination with hematoxylin, eosin and Giemsa revealed diffuse lymphohistiocytic dermal infiltration with intracytoplasmic amastigotes, arriving at the diagnosis of chagasic hypodermitis. Skin cultures were negative (Figures 3 and 4). She was prescribed benznidazole 160mg bd for 60 days, with resolution of cutaneous lesions and negative blood Chagas PCR.

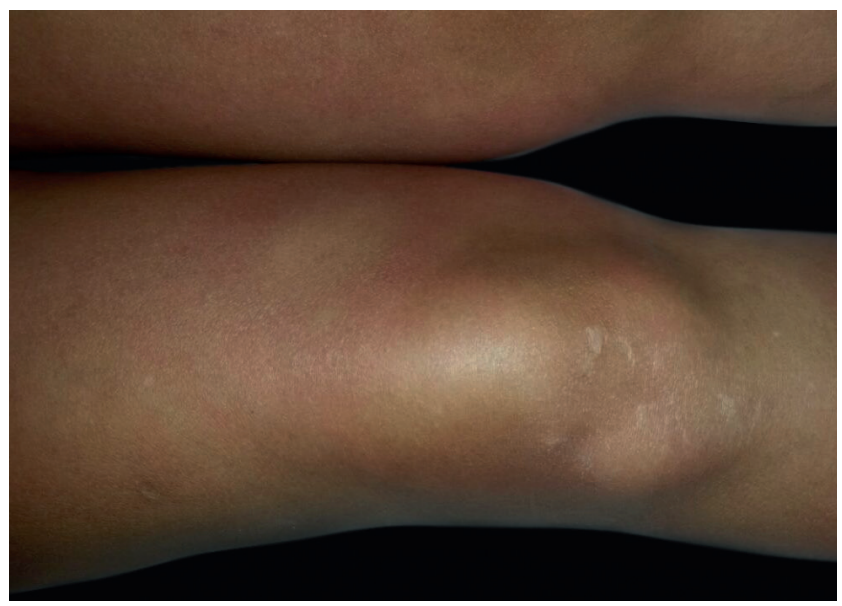

Figure 1: Multiple erythematous, infiltrated plaques, with diffuse borders, irregular size and shape, with increase of the local temperature, extended on both gluteus, thighs and legs

\section{DISCUSSION}

The incidence of reactivation among organ transplant recipients is variable, close to $20 \%$ and can rise to $40 \%$ in allogeneic bone marrow transplant patients. ${ }^{9}$ Because of this, a pre-transplant screening with enzyme immunoassay indirect hemagglutination and indirect immunofluorescence should be performed in all patients native to Latin-American countries, born to Latin-American mothers, recipients of multiple transfusions, or those who have resided or traveled to a endemic area for more than 6 months. A positive diagnosis of infection is reached with two positive tests. ${ }^{4}$

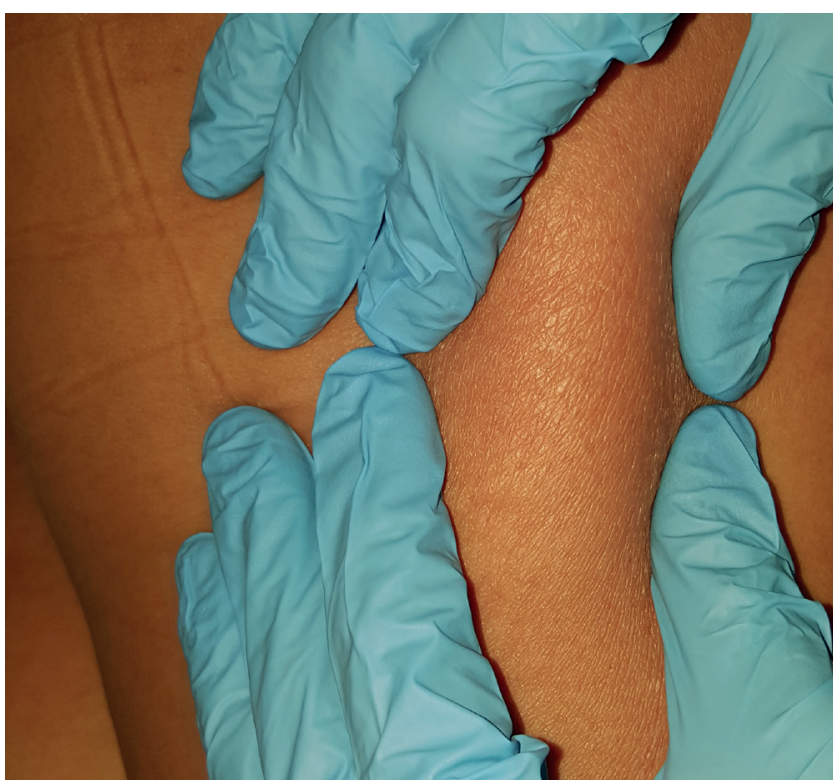

Figure 2: Multiple erythematous, infiltrated plaques, with diffuse borders, irregular size and shape, with increase of the local temperature, extended on both gluteus, thighs and legs

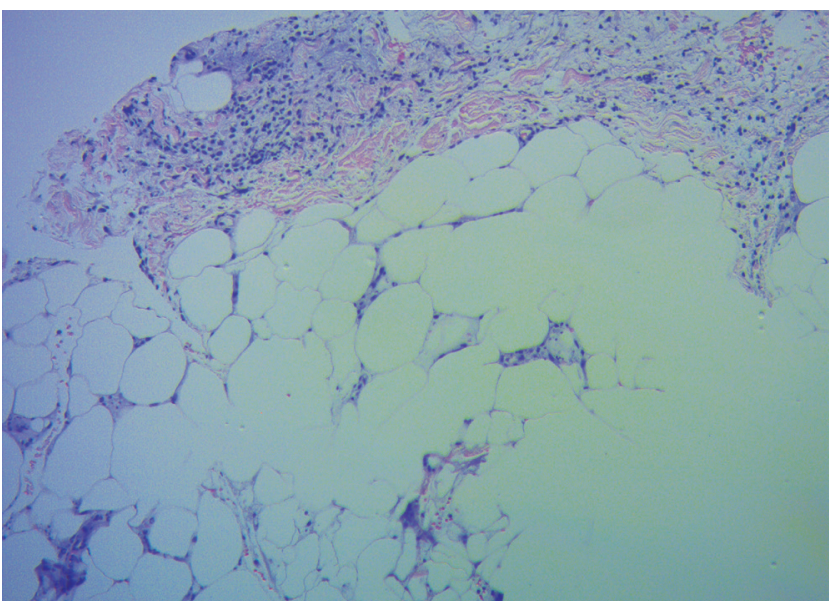

FigURE 3: Skin biopsy revealed diffuse dermal and hypodermal lymphohistiocytic infiltrate with compromise of lobular septa. Hematoxylin \& eosin $(x 10)$ 


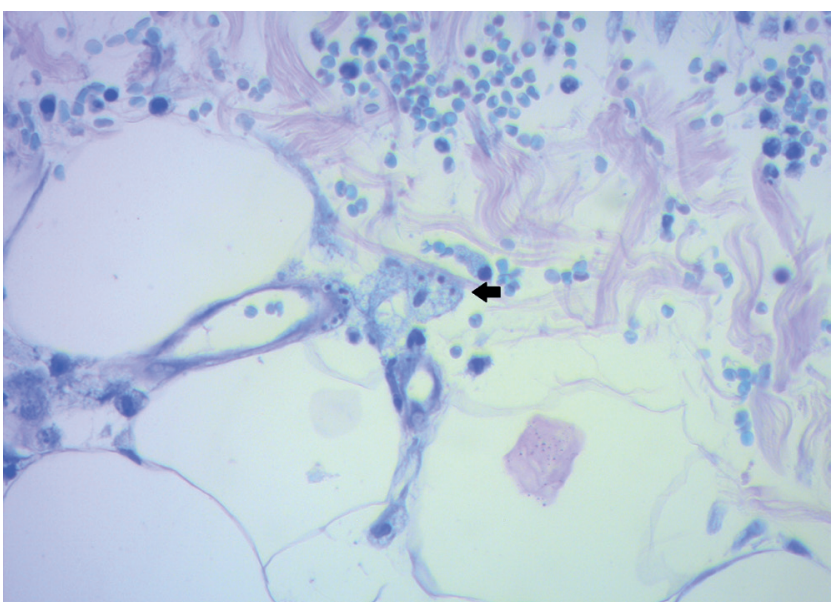

FIGURE 4: Skin biopsy with Giemsa (x40), revealed intracytoplasmic amastigotes

All transplant recipients with positive serology tests should be monitored weekly for the first 6 months, monthly thereafter, and weekly after intensification of immunosuppression for at least 2 months, with direct methods. ${ }^{4}$ This unfortunately was not done adequately in the case mentioned due to lack of connection between the patient and the hospital.

The first line of treatment in acute and reactivated disease is benznidazole in doses of $5 \mathrm{mg} / \mathrm{kg} /$ day. PCR should be performed weekly at least until two negative results are obtained. Follow-up should return to basal schedule of monitoring according to posttransplant time.

Blood counts and hepatic enzymes should also be checked before treatment and 15 to 20 days after initiation because the drug can generate leukopenia, agranulocytosis and hepatotoxicity. The second line of treatment is with $8 \mathrm{mg} / \mathrm{kg} /$ day of nifurtimox and should be reserved for patients with benznidazole side effects or infections with resistant strains. During the use of this drug, the effects on the nervous system must be monitored because it can generate peripheral polyneuritis and/or convulsions, which would force the treatment to be discontinued. Both treatments are suggested to be performed for 30 to 60 days. ${ }^{1,10}$

Chagas' disease is a zoonosis typically associated to Latin American countries and low economic resources. In Argentina, Córdoba represents a medium risk area of transmission. Nowadays, as a consequence of globalization, the disease has spread around the world, which makes it imperative for dermatologists worldwide to be familiar with this entity even in patients who do not come from endemic areas. The high incidence of transplants and the extended use of immunosuppressive treatment generate a susceptible population to suffer from Chagas reactivation. It is essential, therefore, to perform the appropriate serological tests in the pre-transplant evaluation of those patients who have indication, and a close post-transplant follow-up of those who have chronic disease. We reinforce the importance of the role of the medical team in maintaining a high clinical suspicion, in patients who come from an endemic and non-endemic area, which will determine an early diagnosis of the disease, adequate treatment and prevention of complications. $\square$

\section{REFERENCES}

1. Hemmige V, Tanowitz H, Sethi A.Trypanosoma cruzi infection: a review with emphasis on cutaneous manifestations. Int J Dermatol. 2012:51:501-8.

2. Msal.gob.ar [Internet]. Ministerio de Salud de la Nación. Buenos Aires: Ministerio de Salud de la Nación, 2012. Guías para la atención al paciente infectado con Trypanosoma cruzi (Enfermedad de Chagas) [cited 2018 Feb 08]. Available from: http://www.msal.gob.ar/chagas/images/stories/Equipos/Guia_Nacional_Chagas_ version 27092012.pdf.

3. La Forgia MP, Pellerano G, de las Mercedes Portaluppi M, Kien MC, Chouela EN. Cutaneous manifestation of reactivation of Chagas disease in a renal transplant patient: long-term follow-up. Arch Dermatol. 2003;139:104-5.

4. Chagas' Disease Argentine Collaborative Transplant Consortium, Casadei D. Chagas' disease and solid organ transplantation. Transplant Proc. 2010;42:3354-9.

5. Gallerano V, Consigli J, Pereyra S, Gómez Zanni S, Danielo C, Gallerano RH, et

al. Chagas' disease reactivation with skin symptoms in a patient with kidney transplant. Int J Dermatol. 2007;46:607-10.

6. Apt BW, Heitmann IG, Jercic MIL, Jofré ML, Muñoz CVP, Noemí HI, et al . Guías clínicas de la enfermedad de Chagas: Parte IV. Enfermedad de Chagas en pacientes inmunocomprometidos. Rev Chil Infectol. 2008;25:289-92.

7. Altclas J, Sinagra A, Jaimovich G, Salgueira C, Luna C, Requejo A, et al. Reactivation of chronic Chagas' disease following allogeneic bone marrow transplantation and successful pre-emptive therapy with benznidazole. Transp Infect Dis. 1999:1:135-7.

8. Navarro Tuculet C, Hidalgo Parra I, Enz P, Galimberti G, Galimberti RL. Chagas disease: cutaneous manifestation of a patient with liver transplantation. Reactivation. Cutan Iber Lat Am. 2007;35:25-8

9. Riganti J, Maqueda MG, Piñero MC, Volonteri VI, Galimberti RL. Reactivation of Chagas' disease: cutaneous manifestations in two immunosuppressed patients. Int J Dermatol. 2012;51:829-34.

10. Riarte A1, Luna C, Sabatiello R, Sinagra A, Schiavelli R, De Rissio A, et al. Chagas' disease in patients with kidney transplants: 7 years of experience 1989-1996. Clin Infect Dis. 1999;29:561-7.

AUTHORS'CONTRIBUTIONS

Maria Guillermina Ferraresso $\quad$ (iD) ORCID 0000-0002-2672-8775

Elaboration and writing of the manuscript, Obtaining, analyzing and interpreting the data, Effective participation in research orientation, Critical review of the literature, Critical review of the manuscript

Ana Clara Torre $\quad$ (iD) ORCID 0000-0001-9670-6566

Approval of the final version of the manuscript, Conception and planning of the study, Elaboration and writing of the manuscript, Obtaining, analyzing and interpreting the data, Effective participation in research orientation, Intellectual participation in propaedeutic and/or therapeutic conduct of the cases studied, Critical review of the literature, Critical review of the manuscript

Maria Manuela Martínez Piva $\quad$ (iD) ORCID 0000-0003-1007-5658

Elaboration and writing of the manuscript, Obtaining, analyzing and interpreting the data, Intellectual participation in propaedeutic and/or therapeutic conduct of the cases studied, Critical review of the literature

Laura Barcan $\quad$ (iD) ORCID 0000-0002-3350-1042

Approval of the final version of the manuscript, Elaboration and writing of the manuscript, Intellectual participation in propaedeutic and/or therapeutic conduct of the cases studied, Critical review of the literature, Critical review of the manuscript

How to cite this article: Ferraresso MG, Torre AC, Martínez Piva MM, Barcan L. Chagas disease reactivation: cutaneous manifestations in a transplanted patient. An Bras Dermatol. 2018;93(6):890-2. 